\title{
Hyperhidrose
}

\section{Nasse Stellen trocken legen}

Schwitzen ist lebenswichtig und schützt den Körper vor Überhitzung. Rinnt der Schweiß aber ganz ohne Grund, macht das Probleme. Schwitzen ist nicht salonfähig, und sichtbare Schweißflecken unter der Achsel sind ebenso unangenehm wie ein feuchter Händedruck. Glücklicherweise kann den Betroffenen geholfen werden.

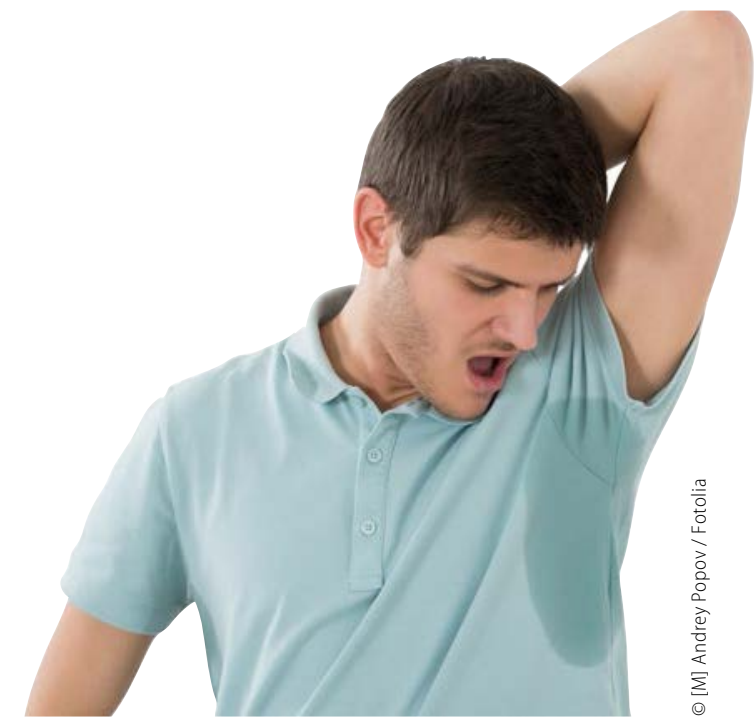

Gemäß der aktuellen Leitlinie ist für die Diagnose „Hyperhidrose“ nicht die absolute Schweißmenge entscheidend, sondern das inadäquate Schwitzen. Entsprechend gibt es keine allgemeingültigen Mess- oder Laborwerte, anhand derer eine Hyperhidrose diagnostiziert oder ausgeschlossen werden kann. Vielmehr sind die Anamnese und das klinische Bild ausschlaggebend.

Bei der primären idiopathischen Hyperhidrose lassen sich - anders als bei der sekundären - keine internistischen Erkrankungen oder externen Ursachen eruieren. Meist tritt sie fokal begrenzt auf, bevorzugt an Achseln, Händen, Füßen, Stirn oder behaarter Kopfhaut. Das Schwitzen beginnt plötzlich und aus dem Nichts heraus. Bei Hyperhidrosepatienten finden sich weder mehr noch größere ekkrine Schweißdrüsen, sie sind lediglich überstimuliert [1].

\section{Behandlung nach Stufenschema}

Ist eine sekundäre Form sicher auszuschließen, kann mit der Behandlung begonnen werden. Bei der Therapie orientiert man sich an einem Stufenplan, wobei gemäß aktueller Leitlinie eine auf den Patienten und die Lokalisation abgestimmte Behandlung zu wählen ist.

\section{Die Drüsenausgänge verschließen}

Bei der palmoplantaren wie auch axillären Hyperhidrose können, wenn sie nur moderat ausgeprägt sind, Antiperspiranzien auf Basis von Aluminiumchloridhexahydrat, anderen Aluminiumsalzen oder gerbsäurehaltigen Topika versucht werden. Die Aluminiumsalze verschließen die Ausführungsgänge der Schweißdrüsen, indem sie in den Drüsengang diffundieren und die Metallionen Komplexe mit den Mukopolysacchariden bilden. Die Leitlinien empfehlen eine Aluminiumchloridhexahydratkonzentration von 10 bis $30 \%$. Das Antiperspiranz ist über mehrere Wochen hinweg abends aufzutragen. Zwar ist die Anwendung zeitlich begrenzt, dennoch darf nicht unerwähnt bleiben, dass das Bundesinstitut für Risikobewertung (BfR) eine gesundheitliche Beeinträchtigung bei der Verwendung von Antitranspiranzien mit einer Aluminiumkonzentration um die $20 \%$ für „möglich“ hält. Die Aussagekraft der vorliegenden Daten schätzt das BfR jedoch als "gering“ ein. Die Kosmetikwissenschaftlerin Meike Streker aus Hamburg empfiehlt, die Aluminiumkonzentration nicht allzu hoch zu wählen und die Antiperspiranzien nur auf gesunde Haut aufzutragen [2].

\section{Kurieren mit Strom}

Die Leitungswasseriontophorese ist die zweite Stufe der Behandlung und gilt bei der palmoplantaren Hyperhidrose als Standardtherapie [3]. An den Achseln ist sie deutlich schwieriger durchzuführen.

\section{Schweißdrüsen lähmen}

Die Chemodenervierung mit Botulinumtoxin A, die dritte Therapiestufe, gilt derzeit unter den konservativen Behandlungsmethoden als die effektivste und ist zumindest an der Achselhöhle leicht anzuwenden [4]. An den Hand- und Fußflächen sind die intrakutanen Injektionen hingegen ziemlich schmerzhaft. Das Neurotoxin blockiert die autonomen cholinergen postganglionären Nervenfasern, sodass die Schweißproduktion sistiert. Nach etwa sechs Monaten lässt die Wirkung jedoch nach und die Behandlung muss wiederholt werden.

\section{Therapie von innen}

Die systemische Behandlung ist v.a. bei generalisierter Hyperhidrose angezeigt, in Einzelfällen empfiehlt sie die Leitlinie auch bei therapieresistenten lokalisierten Formen. Zugelassen sind in Deutschland für diese Indikation zwei anticholinerg wirksame Präparate. Allerdings sind die möglichen unerwünschten Nebenwirkungen wie Akkomodationsstörungen, Mundtrockenheit, Tachykardien, Miktionsstörungen und Konzentrationsstörungen für die Patienten sehr belastend.

In kleineren Fallserien wurden auch Psychopharmaka, Tranquilizer, Sedativa und Betablocker eingesetzt, kontrollierte Studien fehlen aber bislang. Stehen psychische Probleme im Vordergrund, kann laut Leitlinie deren Einsatz im Einzelfall erwogen werden. Strenggenommen handelt es sich dann aber um eine sekundäre Hyperhidrose in Folge einer psychischen Grunderkrankung.

\section{Manchmal hilft nur die Operation}

Bringen konservative Maßnahmen nicht den gewünschten Erfolg und ist der Leidensdruck hoch, kommen auch operative Maßnahmen infrage. In der Achselhöhle lassen sich die Schweißdrüsen relativ einfach entfernen. Die endoskopische thorakale Sympathektomie wiederum kann als Ultima Ratio eingesetzt werden, wenn das Schwitzen an den Händen anders nicht in den Griff zu bekommen ist. Von der lumbalen Sympathektomie hingegen wird eher abgeraten [3].

(Dagmar Kraus)

\footnotetext{
1. Leitlinie "Definition und Therapie der primären Hyperhidrose" Registernummer 013/059

2. hautnah 2014;13:10-11

3. Hölzle E. et al. Hautarzt 2012;63:448-451

4. Rapprich S. ästhetische dermatologie 2014;6:30-38
} 